\title{
ASSOCLAÇĀO ENTRE ACHADOS ABREUGRÁFICOS ANÔMALOS DO APARELHO RESPIRATÓRIO E MANIFESTAÇÕES CLÍNICAS
}

\author{
João Claudio Flores-Cardoso \\ Afonso Dinis Costa-Passos" \\ Antonio Ruffino-Netto
}

\begin{abstract}
FLORES-CARDOSO, J.C. et al. Associação entre achados abreugráficos anômalos do aparelho respiratório e manifestaçóes clínicas. Rev. Saúde públ., S. Paulo, 23: 368-73, 1989.

RESUMO: Foram analisados os registros dos principais sintomas de anamnese do aparelho respiratório e de diagnóstico de pneumopatia, nas histórias clínicas de primeira consulta de pacientes com achados anormais do aparelho respiratório na abreugrafia de rotina realizada no Hospital das Clínicas da Faculdade de Medicina de Ribeirăo Preto, a partir de 1967. Foram tomados 997 controles (pacientes sem alteraçăo na abreugrafia) pareados com os casos segundo idade e sexo. Em cerca de $1 / 3$ do total de observaçóes clínicas nåo foi registrada anamnese do aparelho respiratório. Verificou-se major proporçăo de registro de sintomas respiratórios e de diagnóstico de pneumopatia entre os casos que entre os controles. O mesmo achado foi verificado para os casos quando os mesmos foram separados em dois grupos de acordo com a maior ou menor gravidade das lesøes apresentadas. Hemoptise e dor torácica loram os sintomas que mclhor discriminaram doentes de sadios. Recomenda-se que o uso da abreugrafia para diagnóstico de alterą̧óes respiratórias seja seletivo, aplicando-se apenas a pacientes com queixas de hemoptise, dor torácica e tosse e/ou expectoração, isoladamente ou combinadas.
\end{abstract}

DESCRITORES: Radiografia pulmonar de massa, utilizaçăo. Pneumopatias, prevençăo. Hemoptise. Dor no peito. Tosse.

\section{INTRODUÇÃo}

Desde o seu desenvolvimento por Manoel de Abreu, em 1936, a abreugrafla passou a ser utillzada como meio prático e de baixo custo para a realização de radiografias periódicas e em série, visando, principalmente, o diagnóstico precoce de tuberculose pulmonar. A estreita vinculaçāo que se estabeleceu entre esta doença e o novo método diagnóstico acabou por relegar a um segundo plano a sua capacidade de evidenciar outras lesōes e afecçōes, tais como câncer e outros tumores torácicos, processos pleurais, enfisema, doenças pulmonares micóticas, metástases e lesōes cardiovasculares. À exceçāo do câncer pulmonar, são muito raras as referências relativas às vantagens da abreugrafia no diagnóstico precoce de outras patologias que não seja a tuberculose pulmonar, limitando-se geralmente os trabalhos à uma mera citação das condições que podem ser detectadas a partir do exame $\mathrm{e}^{3,10}$.

Entre 1962 e 1979, o Hospital das Clínicas da Faculdade de Medicina de Ribeirāo Preto mante- ve como rotina abreugrafar todos os pacientes que o procuravam pela primeira vez, exceto gestantes e menores de 15 anos de idade. A necessidade de avaliar a eficácia e a eflciência deste procedimento motivou a execução de duas investigaçōes retros. pectivas $^{7,9}$, tendo uma delas - referente ao estudo de associação entre achados abreugráficos de cardiomegalia e manifestaçōes clínicas - sido objeto de publicaçāo anterior ${ }^{8}$.

$O$ presente trabalho constitui parte da segunda investigaçāo e tem como objetivo estudar a associaçāo entre achados abreugráficos pulmonares anômalos e: a) sintomas respiratórios registrados nos prontuários médicos de um hospital universitário; b) diagnóstico formulado na primeira consulta.

\section{MATERIAL E MÉTODO}

A partir da revisão dos livros do Serviço de Abreugrafia foram selecionados, como casos, todos os pacientes com registro de qualquer alteração abreugráfica do aparelho respiratório ao pro-

\footnotetext{
* Departamento de Saúde Comunitária do Centro de Ciencias Médicas e Biológicas da Universidade Federal de Sergipe Hospital Universitário - Bairro Santo Antonio - 49.000 - Aracaju, SE - Brasil.

** Departamento de Medicina Social da Faculdade de Medicina de Ribeiråo Preto da Universidade de Săo Paulo - Av. Bandeirantes, 3900 - 14049 - Ribeirăo Preto, SP - Brasil.
} 
curarem o Hospital das Clínicas pela primeira vez, entre 25/9/1967 e 25/10/1969. As allerações radiológicas foram classificadas scyundo uma adaptação da "Sistematização das Imingens Patológicas do Tórax"4, reunindo as imagens em quatro grupos: A - Imagens de hipotransparência; B Imagens de hipertransparência; C - Outras imagens; D - Imagens não especificadas na classificação.

Para cada caso foi selecionado um controle, representado pelo paciente imediatamente seguinte ao caso no livro de registro, que tivesse laudo abreugráfico normal e que satisfizesse os critérios de pareamento. Estes, incluíram apenas as variáveis sexo e idade, a qual foi considerada nas faixas de $15-24,25-34,35-44,45-54$ e 55 e + anos completos. Também as variáveis cor e estado civil foram obtidas a partir do mesmo registro.

A seguir foram revisados os prontuários dos casos e dos controles, preenchendo-se para cada um deles uma ficha específica, na qual se registraram as seguintes variáveis: profissão, procedência, clínica do hospital onde ocorreu a primeira consulta, tipo de queixa respiratória relatada (dispnéia, tosse e/ou expectoração, hemoptise e dor torácica, exceto a dor caracterizada como angina pectoris). A revisão dos prontuários e a transcrição das informações para a ficha específica foram realizadas por uma única pessoa, com um tempo médio semelhante para o preenchimento das fichas dos casos e dos controles.

Foi considerada a presença de queixa correlata sempre que o paciente referia ao menos uma das queixas respiratórias acima citadas, classificadas em:

\section{A - Registrada}

A1 - Presente - quando sua presença foi registrada na história clínica, seja na queixa principal, história da moléstia atual ou no interrogatório sobre os diversos aparelhos.

A2 - Negada - quando a ausência da queixa foi registrada na história clínica (queixa principal, história da moléstia atual ou interrogatório sobre os diversos aparelhos). Foi considerado suficiente o registro de ausência de uma das queixas.

\section{B - Não Registrada}

Quando a história clínica não registrava a presença nem a ausência das queixas.
De acordo com as imagens abreugráficas anômalas de que fossem portadores, os pacientes foram classificados em dois grupos, segundo a menor ou maior probabilidade de apresentar sintomas:

Grupo S- : Indivíduos com menor probabilidade de apresentar sintomatologia respiratória, correspondendo a abreugrafias com um dos seguintes tipos de imagens: micronódulos, macronódulos, fibrose, espessamento pleural e de fissuras, alterações diafragmáticas e o grupo de lesões não enquadradas na classificação.

Grupo S + : Individuos com maior probabilidade de apresentar sintomatologia respiratória, correspondendo a abreugrafias com imagens de um ou mais dos seguintes tipos: velamento, infiltrado, enfisema, imagens flocosas, calcificaçōes nāo nodulares, bronquiectasias e associações do grupo $\mathrm{S}+\mathrm{e} \mathrm{S}$ -

Para análise estatística foi utilizado o teste qui-quadrado.

\section{RESULTADOS}

A Tabela 1 mostra a distribuição dos casos e controles segundo o sexo e o grupo etário. Observa-se que no sexo masculino ocorre aumento constante do número de casos em função da idade. Entre as mulheres verifica-se uma tendência semelhante, porém com uma súbita interrupção na faixa de 25-35 anos, que participa com o menor número de indivíduos (7,8\%). Obviamente, a distribuição é idêntica para os mesmos grupos etários e sexo, tanto nos casos como nos controles, em função do pareamento efetuado (pareamento individual, com razão de pareamento igual a 1).

Na Tabela 2 é apresentada a distribuição do registro de queixas entre casos e controles. A presença de queixas foi mais freqũente nos casos $(36,9 \%)$ que no grupo controle $(22,4 \%)$, ocorrendo o oposto em relação à ausência das mesmas $(32,7 \%$ e $39,6 \%$, respectivamente). A freqüência de subregistro foi elevada em ambos os grupos $(30,4 \%$ e $38,0 \%)$. Analisando-se apenas o registro da presença ou ausência de queixas, observa-se diferença estatisticamente significativa $\left(X_{1}^{2} G L=\right.$ 37,$9 ; p<0,001$ ).

A distribuição das queixas nos dois grupos (Tabela 3) mostra um total duas vezes maior entre os casos que entre os controles ( 577 e 286), representando o acometimento de $57,9 \%$ e $28,7 \%$ de cada um destes grupos, respectivamente. Dispnéia e tosse e/ou expectoraçāo predominaram amplamente nos dois grupos, com proporçōes mais altas 
TABELA 1

Distribuição dos casos e controles segundo o sexo e o grupo etário

\begin{tabular}{|c|c|c|c|c|c|c|c|c|c|c|}
\hline \multirow{3}{*}{$\begin{array}{l}\text { Grupo } \\
\text { Etário }\end{array}$} & \multicolumn{4}{|c|}{ Casos } & \multicolumn{4}{|c|}{ Controles } & \multirow{2}{*}{\multicolumn{2}{|c|}{ Total }} \\
\hline & \multicolumn{2}{|c|}{ Masculino } & \multicolumn{2}{|c|}{ Feminino } & \multicolumn{2}{|c|}{ Masculino } & \multicolumn{2}{|c|}{ Feminino } & & \\
\hline & № & $\%$ & No & $\%$ & No & $\%$ & No & $\%$ & No & $\%$ \\
\hline $\begin{array}{l}15-25 \\
25-35 \\
35-45 \\
45-\end{array}$ & $\begin{array}{r}49 \\
68 \\
89 \\
370\end{array}$ & $\begin{array}{r}8,5 \\
11,8 \\
15,5 \\
64,2\end{array}$ & $\begin{array}{r}60 \\
33 \\
87 \\
241\end{array}$ & $\begin{array}{r}14,3 \\
7,8 \\
20,7 \\
57,2\end{array}$ & $\begin{array}{r}49 \\
68 \\
89 \\
370\end{array}$ & $\begin{array}{r}8,5 \\
11,8 \\
15,5 \\
64,2\end{array}$ & $\begin{array}{r}60 \\
33 \\
87 \\
241\end{array}$ & $\begin{array}{r}14,3 \\
7,8 \\
20,7 \\
57,2\end{array}$ & $\begin{array}{r}218 \\
202 \\
352 \\
1.222\end{array}$ & $\begin{array}{l}10,9 \\
10,1 \\
17,7 \\
61,3\end{array}$ \\
\hline Total & 576 & 100,0 & 421 & 100,0 & 576 & 100,0 & 421 & 100,0 & 1.994 & 100,0 \\
\hline
\end{tabular}

TABELA 2

Distribuição dos casos e controles segundo o tipo de registro de queixa respiratória na história clínica da primeira consulta.

\begin{tabular}{|c|c|c|c|c|c|c|}
\hline \multirow{2}{*}{$\begin{array}{c}\text { Registro } \\
\text { de } \\
\text { queixa }\end{array}$} & \multicolumn{2}{|c|}{ Casos } & \multicolumn{2}{|c|}{ Controles } & \multicolumn{2}{|c|}{ Total } \\
\hline & No' & $\%$ & No & $\%$ & Nọ & $\%$ \\
\hline \multicolumn{7}{|l|}{ Registrada } \\
\hline $\begin{array}{l}\text { Presen te } \\
\text { Negada }\end{array}$ & $\begin{array}{l}368 \\
326\end{array}$ & $\begin{array}{l}36,9 \\
32,7\end{array}$ & $\begin{array}{l}223 \\
395\end{array}$ & $\begin{array}{l}22,4 \\
39,6\end{array}$ & $\begin{array}{l}591 \\
721\end{array}$ & $\begin{array}{l}29,6 \\
36,2\end{array}$ \\
\hline Não registrada & 303 & 30,4 & 379 & 38,0 & 682 & 34,2 \\
\hline Total & 997 & 100,0 & 997 & 100,0 & 1.994 & 100,0 \\
\hline
\end{tabular}

Considerando apenas queixas presentes e negadas:

$\mathrm{p}<0,001$

entre os casos. Dor torácica e, especialmente hemoptise, foram acentuadamente mais comuns entre os casos, com uma relação entre os grupos igual a 3,8 para dor torácica $(6,9 \%$ e $1,8 \%)$ e 22,0 para hemoptise $(4,4 \%$ e $0,2 \%)$. A análise estatística mostra diferença significativa $\left(X_{3}^{2} \mathrm{GL}=33,7 ; \mathrm{p}<\right.$ 0,001 ).

A Tabela 4 mostra os casos e controles segundo o número de queixas presentes na primeira consulta. Verifica-se um predomínio progressivamente maior do número absoluto de casos, em relação aos controles, à medida que aumenta o número de queixas, passando de uma relação de 1,3 para 2,2 e 4,7 quando se considera a presença de uma, duas e três queixas, respectivamente. Concomitância de 4 queixas foi observada em 11 casos $(3,0 \%)$ e em nenhum controle. Em termos proporcionais, dentro de cada grupo, a presença de uma única queixa foi mais freqüente entre os controles, invertendo-se esta relação a partir da presença de duas queixas ou mais. As diferenças mostram-se estatisticamente significativas $\left(\mathrm{X}_{3}^{2} \mathrm{GL}\right.$ $=23,0 ; p<0,001$ ).
TABELA 3

Distribuição das queixas respiratórias nos casos e controles, segundo tipo

\begin{tabular}{|c|c|c|c|c|}
\hline \multirow{2}{*}{$\begin{array}{l}\text { Tipo de } \\
\text { queixa } \\
\text { respiratória }\end{array}$} & \multicolumn{2}{|c|}{ Casos } & \multicolumn{2}{|c|}{ Controles } \\
\hline & No & $\% *$ & N? & $\% *$ \\
\hline Dispnéia & 242 & 24,3 & 165 & 16,5 \\
\hline Tosse e/ou expectoração & 222 & 22,3 & 101 & 10,1 \\
\hline Hemoptise & 44 & 4,4 & 2 & 0,2 \\
\hline Dor torácica & 69 & 6,9 & 18 & 1,8 \\
\hline Total & 577 & 57,9 & 286 & 28,7 \\
\hline
\end{tabular}

* Em relação ao total de casos e de controles $\mathrm{p}<0,001$

$\mathrm{O}$ grupo de indivíduos com imagens indicativas de lesōes respiratórias mais graves $(S+)$ apresentou um número maior de registro de queixas (tanto presentes como negadas) na primeira consulta, quando comparado ao grupo com lesões mais leves (Tabela 5). Os dois grupos mostraram 
TABELA 4

Distribuição dos casos e controles segundo o número de queixas presentes na história clínica da primeira consulta

\begin{tabular}{crrrrr}
\hline \multirow{2}{*}{$\begin{array}{c}\text { Número } \\
\text { de } \\
\text { queixas }\end{array}$} & No & $\%$ & & No & $\%$ \\
\cline { 2 - 3 } \cline { 6 - 6 } \cline { 5 - 6 } & 214 & 58,2 & & 167 & 74,9 \\
2 & 110 & 29,9 & & 49 & 22,0 \\
3 & 33 & 9,0 & & 7 & 3,1 \\
4 & 11 & 3,0 & & - \\
\hline Total & 368 & 100,0 & 223 & 100,0 \\
\hline
\end{tabular}

$\mathrm{p}<0,001$

distribuições proporcionais diferentes de registro de queixas presentes e negadas, com predomínio das primeiras no $\mathrm{S}+\mathrm{e}$ das últimas no $\mathrm{S}-$. A análise estatística mostra diferença significativa $\left(\mathrm{X}_{1}^{2} \mathrm{GL}=\right.$ 7,17; $\mathrm{p}<0,01)$.

Na Tabela 6 é apresentada a distribuição dos casos e controles segundo o registro de diagnóstico de pneumopatia na primeira consulta. Verifica-se que o diagnóstico foi registrado como presente em $11,0 \%$ dos casos e em apenas $1,7 \%$ dos controles, resultando um risco adicional ("odds ratio") igual a $7,1\left(X_{1}^{2} \mathrm{GL}=72,7 ; p<0,001\right)$.

Na Tabela 7 observa-se que também os indivíduos do grupo $S+$ apresentaram uma proporção significativamente mais alta de registro de diagnóstico de pneumopatia quando comparados aos do grupo $S$-, com valores de $14,2 \%$ e $4,3 \%$ respectivamente. $\mathrm{O}$ risco adicional ("odds ratio") mostra um valor de $3,6\left(X_{1}^{2} \mathrm{GL}=21,6 ; \mathrm{p}<0,001\right)$.

\section{TABELA 5}

Distribuição dos casos por grupos de imagens abreugráficas anômalas, segundo o registro da presença ou ausência de queixa respiratória na primeira consulta.

\begin{tabular}{|c|c|c|c|c|}
\hline \multirow{3}{*}{ Queixa } & \multicolumn{4}{|c|}{ Grupos de Imagens Anômalas } \\
\hline & \multicolumn{2}{|c|}{$S+$} & \multicolumn{2}{|c|}{$S-$} \\
\hline & No & $\%$ & No & $\%$ \\
\hline $\begin{array}{l}\text { Presente } \\
\text { Negada }\end{array}$ & $\begin{array}{l}266 \\
203\end{array}$ & $\begin{array}{l}56,7 \\
43,3\end{array}$ & $\begin{array}{l}102 \\
122\end{array}$ & $\begin{array}{l}45,5 \\
54,5\end{array}$ \\
\hline Total & 469 & 100,0 & 224 & 100,0 \\
\hline
\end{tabular}

$\mathrm{p}<0,01$
TABELA 6

Distribuição dos casos e controles segundo o registro ou não de diagnóstico de pneumopatia na impressão diagnóstica da primeira consulta

\begin{tabular}{llllll}
\hline $\begin{array}{l}\text { Registro de } \\
\text { diagnóstico }\end{array}$ & \multicolumn{2}{c}{ Casos } & & \multicolumn{2}{c}{ Controles } \\
\cline { 2 - 3 } \cline { 6 - 6 } de pneumopatia & No & $\%$ & & No & $\%$ \\
\hline Presente & 110 & 11,0 & & 17 & 1,7 \\
Ausente & 887 & 89,0 & 980 & 98,3 \\
\hline Total & 977 & 100,0 & & 997 & 100,0 \\
\hline
\end{tabular}

$\mathrm{p}<0,001$

TABELA 7

Disțribuição dos grupos de imagens abreugráficas anômalas, segundo o tipo de diagnóstico registrado na primeira consulta.

\begin{tabular}{|c|c|c|c|c|}
\hline \multirow{3}{*}{$\begin{array}{l}\text { Diagnóstico(s) } \\
\text { registrado(s) }\end{array}$} & \multicolumn{4}{|c|}{ Grupos de Imagens Anômalas } \\
\hline & \multicolumn{2}{|c|}{$S+$} & \multicolumn{2}{|c|}{$S-$} \\
\hline & No & $\%$ & Nọ & $\%$ \\
\hline $\begin{array}{l}\text { Pneumopatia(s) } \\
\text { Outro(s) }\end{array}$ & $\begin{array}{r}96 \\
579\end{array}$ & $\begin{array}{l}14,2 \\
85,8\end{array}$ & $\begin{array}{r}14 \\
308\end{array}$ & $\begin{array}{r}4,3 \\
95,7\end{array}$ \\
\hline Total & 675 & 100,0 & 322 & 100,0 \\
\hline
\end{tabular}

$\mathrm{p}<0,001$

\section{DISCUSSĀO E CONCLUSÓES}

Muito embora exames abreugráficos continuem sendo muito utilizados pelos serviços de saúde, chama a atenção a relativa escassez de publicações que se preocupam em avaliar os benefícios advindos desta utilização em larga escala. Em nosso meio, algumas investigações têm abordado o assunto, embora quase todas com ênfase na avaliaçāo do método quando aplicado ao diagnóstico de tuberculose pulmonar ${ }^{1,2,3}$. Em artigo recentemente publicado 8 , desenvolvido com metodologia semelhante ao presente estudo, foi avaliada a utilização da abreugrafia na detecção de alteraçōes cardiocirculatórias no Hospital das Clínicas da Faculdade de Medicina de Ribeirão Preto (HC/FMRP).

Considerando-se apenas a população acima de 15 anos de idade, a demanda do HC/FMRP é representada na sua maior parte por indivíduos acima de 35 anos (Tabela 1). A menor participação do sexo feminino na faixa de 25 a 35 anos está provavelmente associada à maior concentração de 
mulheres em idade fértil nestas idades, o que limita o uso da abreugrafia em função da possibilidade maior de gravidez entre essas pacientes.

A avaliação da qualidade das histórias clínicas através da observação de registro ou não de queixas respiratórias (Tabela 2), mostra que em aproximadamente $1 / 3$ das histórias referentes aos casos e em maior proporção nos controles não há registro de anamnese do aparelho respiratório.

Quanto ao diagnóstico de pneumopatia na primeira consulta (Tabela 6), o procedimento utilizado na obtenção desta informação consistiu em procurar o registro da mesma na impressão diagnóstica da história clínica, nāo importando se era diagnóstico principal ou secundário, nem o tipo de pneumopatia diagnosticada. Adotou-se tal critério em virtude da grande subjetividade envolvida nas decisões relativas aos diagnósticos clínicos e sua avaliação. A ocorrência em maior ou menor proporção de sub-registros de sintomas respiratorios e diagnósticos de pneumopatia em histórias clínicas de rotina está associada a múltiplas causas, destacando-se aquelas ligadas aos pacientes, médicos, educação médica e ao tipo de assistência oferecida. Especificamente em relação ao HC/FMRP, a qualidade dos dados de registro já foi objeto de várias publicaçōes anteriores $9,11,12$.

Analisando-se a presença de queixas respiratórias, em geral registradas na primeira consulta (Tabela 2), observa-se que entre os casos elas se fazem presentes em proporçōes mais altas que entre os controles. Ao se particularizar o tipo de queixa, verifica-se que nem todas apresentam a mesma capacidade de discriminar doentes de sadios (Tabela 3). Assim é que dispnéia, embora mais freqüente entre os casos, aparece com alta proporção também como queixa presente entre os controles. Ao estudar, no mesmo hospital e no mesmo período, a associação entre diferentes graus de cardiomegalia evidenciados pela abreugrafia e o registro dos principais sintomas cardiorespiratórios, Costa-Passos ${ }^{\top}$ verificou que a queixa de dispnéia também ocorria em alta proporção entre os pacientes sem aumento da área cardíaca. Uma possível explicação para o achado, em ambos os estudos, seria uma falta de caracterização adequada do sintoma, tanto por parte de médicos como de pacientes, com freqüente registro da queixa sem um correspondente substrato fisiopa- tológico. Adicionalmente, pode ser aventada a hipótese de ocorrência, entre os controles, de indivíduos sintomáticos portadores de patologia respiratória não evidenciada pela abreugrafia, tais como asma brônquica ou enfisema pulmonar em fase inicial. $\mathrm{O}$ achado de tosse e/ou expectoração, mesmo se fazendo presente com relativa freqüência entre os controles, mostra uma capacidade melhor de selecionar indivíduos com alterações radiológicas.

A boa capacidade discriminatória mostrada pelos achados de dor torácica e hemoptise está provavelmente associada à maior objetividade dos mesmos, especialmente hemoptise, à qual se acrescenta o impacto emocional que determina no paciente.

A diferente capacidade de discriminação de cada uma das queixas faz com que a presença combinada das mesmas aumente o poder de diferenciar casos e controles. Com efeito, enquanto o registro isolado de um sintoma ocorre em mais alta proporção entre os controles (Tabela 4), a somatória de duas ou mais queixas mostra-se capaz de separar progressivamente melhor doentes e sadios.

$O$ registro de queixas presentes permite diferenciar não apenas casos e controles mas serve também para discriminar, entre os casos, aqueles com imagens abreugráficas de maior gravidade (Tabela 5).

A ocorrência do diagnóstico de pneumopatia com muito maior freqüência entre casos do que entre controles (Tabela 6) e entre indivíduos com imagens anômalas de maior gravidade (Tabela 7), sugere ser o exame abreugráfico potencialmente útil na descoberta de outras lesōes pulmonares relevantes que não apenas a tuberculose. Os dados do presente trabalho indicam que a utilizaçāo da abreugrafia com esta finalidade deva ser seletiva, sempre precedida de um interrogatório simplificado que investigue a ocorrência de tosse e/ou expectoração, hemoptise e dor torácica, ficando a execução do exame condicionada à presença de uma ou mais dessas queixas. A partir desta sugestão, o Serviço de Abreugrafia modificou a sua rotina de serviço e passou a utilizar o referido processo seletivo, cujos resultados serão oportunamente analisados. 
FLORES-CARDOSO, J.C. et al. [The association between anomalous $\mathrm{X}$-ray findings of the respiratory organs and their clinical manifestation]. Rev. Saúde públ, S. Paulo, 23: 368-73, 1989.

ABSTRACT: A retrospective study of hospital charts was conducted for the purpose of analysing the association of roent-genphotographs obtained routinely at the Hospital das Clinicas da Faculdade de Medicina de Ribeirăo Preto, Brazil, since 1967, with the records of respiratory symptoms and pneumopathic diagnosis found in the medical case histories as from the patients' first clinical out-patient consultation at that hospital. For this purpose, 997 patients with abnormal pulmonary roentgenphotography findings were matched according to sex and age with the same number of controls (normal roentgenphotography). It was observed that in one third of the medical histories there was no record of any anamnesis of the respiratory system. A greater proportion of respiratory symptoms and pneumopathic diagnoses was registered in the case group. The same finding separated the patients into two groups according to the severity of the lesions. Hemoptysis and thoracic pain were the symptoms that better differentiated sick from normal patients. A selective utilization of roentgen. photography is recommended, with the method being applied only to patients presenting hemoptysis, thoracic pain and cough/expectoration, either alone or in combination.

KEYwoRDS: Mass chest X-ray, utilization. Lung diseases, prevention. Hemoptysis. Chest pain. Cough.

\section{REFERENCLAS BIBLIOGRÁFICAS}

1. ARANTES, G.R. Aplicaçăo de modelo matemático visando escolha da melhor combinação de instrumentos para a descoberta e tratamento de casos de tuberculose pulmonar. Rev. Saúde públ, S. Paulo, 12:455-70, 1978.

2. ARANTES, G.R Abreugrafia nos programas de luta antituberculose. Rev. Div. nac. Pneumol sanit, 23:35-41, 1979.

3. ARANTES, G.R. \& RUFFINO-NETTO, A. Busca de casos de tuberculose pulmonar: abreugrafia em sintomáticos respiratórios, seguida de exames bacteriológicos nos suspeitos. Rev. Saúde púbL, S. Paulo, 14:185-93, 1980.

4. BETHLEM, N. Pneumologia. Rio de Janeiro, Atheneu, 1973.

5. BJARTVEIT, K. Mass miniature radiography in Norway, today and in the future. Scand. J. resp. Dis.,80(Suppl.):3142, 1972.

6. COMROE, J.H.; FORSTER, R.E.; DUBOIS, B.A.; BRISCOE, W.A.; CARLSEN, E. The lung. $2^{\text {nd }}$ ed. Chicago, Year Book Medical Publ., 1974.

7. COSTA-PASSOS, A.D. Associaçăo entre achados abreugráficos de cardiomegalia e registro de sinais e/ou sinto. mas cardio-respiratórios nas observaçóes clínicas de um hospital geral. Ribeirăo Preto, 1979. [Dissertaçáo de Mestrado - Faculdade de Medicina de Ribeirăo Preto da USP].

8. COSTA-PASSOS, A.D.; FLORES-CARDOSO, J.C.; RUFFINO-NETTO, A. Associaçăo entre achados abreugráficos de cardiomegalia e manifestaçôes clínicas. Rev. Saúde públ, S. Paulo, $21: 29-36,1987$.

9. FLORES-CARDOSO, J.C. Associaçăo entre achados abreugráficos pulmonares e registro de sintomas respiratórios nas histórias clínicas de um hospital geral. Ribeirăo Preto, 1979. [Dissertaçāo de Mestrado - Faculdade de Medicina de Ribeirăo Preto da USP].

10. RISKA, N. The possibilities of applying mass miniature radiography for public health care. Scand. J. resp. Dis., 80(Suppl.):29-30, 1972.

11. RUFFINO-NETTO, A.; SOUZA, 1.C.; PEREIRA, J.C.; GOMES, U.A.; FRANCO, A.R. Avaliação de um serviço de abreugrafia dentro de um hospital universitário: as. pectos preventivos e de educaçăo médica. Medicina, $\mathbf{R i}$. beirăo Preto, 13:1-10, 1981.

12. YAZLLE ROCHA, J.S.; SIMÖES, BJ.G.; FORSTER, A.C.; TAVARES, C; MELLO, M.L.R; MACEDO, M.C.M. Avaliaçăo da utilizaçăo de exames laboratoriais múltiplos num hospital universitário. Medicina, Ribeirão Preto, 16:53-60, 1983.
Recebido para publicação em 23/3/1988 Reapresentado em 18/7/1989 Aprovado para publicação em 5/8/1989 